\title{
Matroid Constrained Fair Allocation Problem
}

\author{
Arpita Biswas, Siddharth Barman \\ Department of Computer Science and Automation, Indian Institute of Science, \\ Bangalore 560012, India \\ arpitab@iisc.ac.in and barman@iisc.ac.in
}

\section{Introduction}

We consider the problem of allocating a set of indivisible goods among a group of agents under matroid constraints and additive valuations, in a fair manner.

The problem of fair allocation of indivisible goods has recently garnered a lot of interest (Amanatidis, Birmpas, and Markakis 2018; Barman et al. 2018; Endriss 2017; Brandt et al. 2016; Amanatidis et al. 2015; Bouveret and Lemaître 2014; Procaccia and Wang 2014; Kurokawa, Procaccia, and Wang 2016). Several important practical problems-for eg., matching courses, resolving inheritance issues, allocating cloud computing resources-naturally lend themselves to the problem of assigning goods to agents, when the goods cannot be fractionally allocated. Significant effort has been invested in devising efficient algorithms for these problems in order to enable practicable solutions to a wide range of such real-world problems.

One of the classical fairness notions for these problems is envy-freeness (EF), which guarantees that no agent values any other agent's bundle more than her own bundle (Foley 1967; Varian 1974). However, EF does not translate to the indivisible scenario, for example, it is impossible to achieve an EF allocation with one indivisible good and two agents. Thus, the challenges pertinent to the fair allocation problem with indivisible goods are (1) defining appropriate fairness notions, (2) providing existential guarantees, and (3) designing computationally efficient algorithms.

A widely used fairness notion for the indivisible setting is envy-freeness up to one good (EF1) (Budish 2011), which is a weaker version of EF. EF1 guarantees that every agent values her bundle at least as much as any other agent's bundle, up to the removal of a single good from the other agent's bundle. Unlike EF, EF1 allocations always exist and can be computed efficiently (Caragiannis et al. 2016). This motivates investigating EF1 under more general settings such as, matroid constraints (Gourvès, Monnot, and Tlilane 2014; Gourvès and Monnot 2017; Biswas and Barman 2018) and connectivity constraints (Bouveret et al. 2017; Bilò et al. 2018). These constraints allow formulating a broader set of interesting and challenging practical problems, see examples provided in (Gourvès, Monnot, and Tlilane 2014;

Copyright (c) 2019, Association for the Advancement of Artificial Intelligence (www.aaai.org). All rights reserved.
Biswas and Barman 2018; Bouveret et al. 2017).

In this paper, we consider the matroid constrained fair allocation problem, introduced in (Biswas and Barman 2018). Here, each allocated bundle, in addition to the fairness criterion, needs to satisfy the independence criterion specified by a matroid. In (Biswas and Barman 2018), the authors established the existence of EF1 allocation for this setting with identical agents and additive valuations; however, designing a polynomial time algorithm remained an open problem. In this paper, we propose a novel algorithm, called SWAP, to augment their existential result.

\section{Preliminaries}

Matroids (Oxley 1992) provide a framework for representing combinatorial constraints-cardinality constraints, laminar constraints etc. Formally, a matroid is defined as a pair $([m], \mathcal{I})$ where $[m]$ is the ground set of elements and $\mathcal{I}$ referred to as a set of independent sets-is a nonempty collection of subsets of $[m]$ that satisfies: (i) Hereditary property: if $B \in \mathcal{I}$ and $A \subset B$, then $A \in \mathcal{I}$, and (ii) Independent Set Exchange: if $A, B \in \mathcal{I}$ and $|A|<|B|$, then there exists an element $x \in B \backslash A$ such that $A \cup\{x\} \in \mathcal{I}$. We consider a fair allocation instance $\left\langle[m],[n],\left(v_{i}\right)_{i \in[n]}, \mathcal{M}\right\rangle$ where $[m]$ is the set of indivisible goods, $[n]$ is the set of agents, $v_{i}(g) \in \mathbb{R}_{+}$denotes the valuation of agent $i \in[n]$ for good $g \in[m]$, and $\mathcal{M}$ denotes the set of all feasible allocations, i.e., whose constituent bundles are independent: $\mathcal{M}:=\{\mathcal{A}$ $\left.=\left(A_{1}, \ldots, A_{n}\right) \in \Pi_{n}([m]) \mid A_{i} \in \mathcal{I}, \forall i \in[n]\right\}$, where $\Pi_{n}([m])$ is the set of all $n$-partitions of the set $[m]$.

\section{The Algorithm: SWAP}

In the unconstrained setting with additive valuations, an EF1 allocation can be obtained using a greedy round robin approach (Caragiannis et al. 2016). However, this approach does not satisfy matroid constraints. Our main contribution is the SWAP algorithm, that finds an EF1 allocation for matroid constrained allocation problems when the valuations are identical $\left(\forall i, j \in[n], v_{i}=v_{j}=v\right)$ and additive $\left(v(S)=\sum_{g \in S} v(g)\right.$ for all $\left.S \subseteq[m]\right)$.

The SWAP algorithm starts with a feasible allocation (not necessarily fair) and intelligently swaps goods among bundles to ensure an EF1 allocation after a finite number of swaps. Our main result is stated in Theorem 1. 
Theorem 1 Given any fair division instance $\left\langle[m],[n],\left(v_{i}\right)_{i \in[n]}, \mathcal{M}\right\rangle$ with additive, identical valuations and matroid constraint, $\mathcal{M} \neq \emptyset$, the algorithm SWAP finds an EF1 allocation in polynomial time.

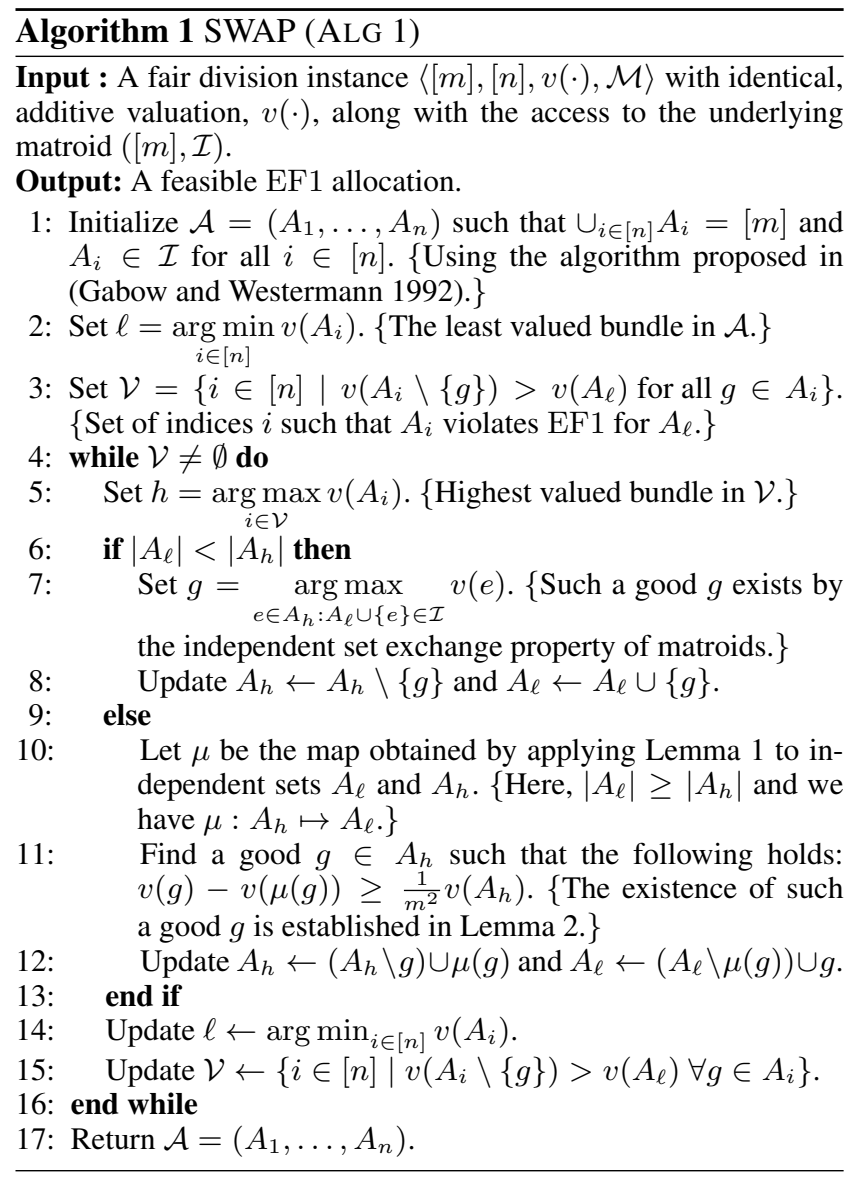

Lemma 1 (Biswas and Barman 2018) Let $([m], \mathcal{I})$ be a matroid with independent subsets $I, J \in \mathcal{I}$ which satisfy $I \cap J=\emptyset$ and $|I| \geq|J|$. Then, there exists a one-to-one map $\mu: J \mapsto I$ such that swapping any $j \in J$ with $\mu(j) \in I$ leads to independent subsets, i.e., for any element $j \in J$, both $(J \backslash\{j\}) \cup \mu(j)$ and $(I \backslash \mu(j)) \cup\{j\}$ belong to $\mathcal{I}$.

Lemma 2 If a feasible allocation $\mathcal{A}$ is not $\mathrm{EF} 1$, then there exists a feasible swap such that the valuation of $A_{h}$-the highest valued bundle violating $\mathrm{EF} 1$ with respect to the least valued bundle-drops by at least a multiplicative factor of $\left(1-\frac{1}{m^{2}}\right)$, or, its cardinality decreases by one.

\section{Conclusions}

This paper strengthens the universality of EF1 by showing that under identical and additive valuations, fair (EF1) allocations can be efficiently computed even under general matroid constraints. Our result provides a computational anchor to the existential result of (Biswas and Barman 2018).

\section{Acknowledgements}

The authors thank Fahad Panolan for useful discussions. Arpita Biswas gratefully acknowledges the support of a Google PhD Fel- lowship Award. Siddharth Barman was supported by a Ramanujan Fellowship (SERB - SB/S2/RJN-128/2015) and a Pratiksha Trust Young Investigator Award.

\section{References}

Amanatidis, G.; Markakis, E.; Nikzad, A.; and Saberi, A. 2015. Approximation Algorithms for Computing Maximin Share Allocations. In International Colloquium on Automata, Languages, and Programming, ICALP, 39-51.

Amanatidis, G.; Birmpas, G.; and Markakis, E. 2018. Comparing Approximate Relaxations of Envy-Freeness. In International Joint Conference on Artificial Intelligence, IJCAI, 42-48.

Barman, S.; Biswas, A.; Krishnamurthy, S. K.; and Narahari, Y. 2018. Groupwise Maximin Fair Allocation of Indivisible Goods. In AAAI Conference on Artificial Intelligence, 917-924.

Bilò, V.; Caragiannis, I.; Flammini, M.; Igarashi, A.; Monaco, G.; Peters, D.; Vinci, C.; and Zwicker, W. S. 2018. Almost envy-free allocations with connected bundles. CoRR abs/1808.09406.

Biswas, A., and Barman, S. 2018. Fair Division Under Cardinality Constraints. In International Joint Conference on Artificial Intelligence IJCAI, 91-97.

Bouveret, S., and Lemaître, M. 2014. Characterizing Conflicts in Fair Division of Indivisible Goods using a Scale of Criteria. In International Conference on Autonomous Agents and Multi-Agent Systems, 1321-1328.

Bouveret, S.; Cechlárová, K.; Elkind, E.; Igarashi, A.; and Peters, D. 2017. Fair Division of a Graph. In International Joint Conference on Artificial Intelligence, IJCAI, 135-141.

Brandt, F.; Conitzer, V.; Endriss, U.; Lang, J.; and Procaccia, A. D. 2016. Handbook of Computational Social Choice. Cambridge University Press.

Budish, E. 2011. The Combinatorial Assignment Problem: Approximate Competitive Equilibrium from Equal Incomes. Journal of Political Economy 119(6):1061-1103.

Caragiannis, I.; Kurokawa, D.; Moulin, H.; Procaccia, A. D.; Shah, N.; and Wang, J. 2016. The Unreasonable Fairness of Maximum Nash Welfare. In ACM Conference on Economics and Computation, EC, 305-322.

Endriss, U. 2017. Trends in Computational Social Choice. Elsevier.

Foley, D. 1967. Resource allocation in the public sector. Yale Economic Essays 7:73-76.

Gabow, H. N., and Westermann, H. H. 1992. Forests, frames, and games: algorithms for matroid sums and applications. Algorithmica 7(1-6):465.

Gourvès, L., and Monnot, J. 2017. Approximate Maximin Share Allocations in Matroids. In International Conference on Algorithms and Complexity, CIAC, 310-321.

Gourvès, L.; Monnot, J.; and Tlilane, L. 2014. Near fairness in matroids. In European Conference on Artificial Intelligence, ECAI, 393-398.

Kurokawa, D.; Procaccia, A. D.; and Wang, J. 2016. When Can the Maximin Share Guarantee Be Guaranteed? In AAAI Conference on Artificial Intelligence, 523-529.

Oxley, J. G. 1992. Matroid Theory.

Procaccia, A. D., and Wang, J. 2014. Fair enough: Guaranteeing approximate maximin shares. In ACM Conference on Economics and Computation, EC, 675-692.

Varian, H. R. 1974. Equity, Envy, and Efficiency. Journal of Economic Theory 9(1):63-91. 